\title{
Grating Couplers For Dual-Channel Thin-Film Waveguide Sensors Produced by Transmission Photolithography
}

\author{
Thomas E. Plowman, ${ }^{\dagger}$ Charles R. Peters, ${ }^{\dagger}$ and W. Monty Reichert ${ }^{\dagger}$ \\ ${ }^{\dagger}$ Duke University, Center for Emerging Cardiovascular Technologies, Department of Biomedical \\ Engineering, Durham, North Carolina 27708; ${ }^{\dagger}$ MCNC Center for Microelectronics, Research Triangle \\ Park, North Carolina 27709 \\ (Paper JBO-133 received Dec. 18 1996; revised manuscript received May 23, 1997; accepted for publication July 4, 1997. )
}

\begin{abstract}
Grating-coupled, thin-film integrated optical waveguide (IOW) structures were fabricated using standard transmission photolithography and employed in a fluoro-affinity assay for the trace detection of analyte. Using a ruled chrome-on-quartz mask with a $0.7 \mu$ repeat, gratings of three etch depths-0.6, 0.8, and 1.0 $\mu \mathrm{m}$-were ion milled into 0.5-mm-thick quartz substrates. Silicon oxynitride (SiON) guiding films $(1.5 \mu \mathrm{m})$ were deposited on the etched substrates by plasma-enhanced chemical vapor deposition (PECVD). Coupling efficiencies for the first diffracted grating orders into the zero-order IOW-guided modes were evaluated at $632.8 \mathrm{~nm}$. The deepest gratings coupled the most light; however, their efficiency was less than half that of prisms. Binding isotherms for fluorescently labeled avidin (Cy5-Av) binding to a biotinylated bovine serum albumin (BSA) adlayer were generated from both prism- and grating-coupled SiON sensor data. Both techniques discriminated the binding of avidin from a $10^{-15} \mathrm{M}$ solution; however run-to-run (intraassay) and between-sensor (interassay) variability reduced reliability of the measurements. () 1997 Society of Photo-Optical Instrumentation Engineers. [S1083-3668(97)00104-4]
\end{abstract}

Keywords planar integrated optical waveguide; photolithography; grating efficiency; affinity sensor.

\section{INTRODUCTION}

Owing to a large interaction path length created by densely packed reflections in the guiding film, thinfilm IOWs are a highly sensitive technique for performing biological assays. Receptor-ligand binding, ${ }^{1}$ protein adsorption, ${ }^{2}$ cell attachment, ${ }^{3}$ molecular diffusion, ${ }^{4}$ and protein hydration ${ }^{5}$ are some of the interfacial events assessed using IOW techniques. More recently, low-loss, sol-gel IOWs produced the first ever broad-band ATR spectrum of an adsorbed protein. ${ }^{6,7} \mathrm{~A}$ comprehensive review of these and other IOW topics is available. ${ }^{8}$

Our prism-coupled, thin films of silicon oxynitride $(\mathrm{SiON})$ allowed fluoroimmunoassay measurements into the femtomolar range. ${ }^{9}$ Although extremely useful in a laboratory setting, the prism coupling of laser light is cumbersome and impractical for the "user friendly" point-of-care device we desire. To alleviate this problem, surface relief gratings have now replaced prisms in our system.

Figure 1 shows the "buried grating" design chosen for use with IOWs. Buried between the substrate and thin film, the grating acts to diffract light into the IOW. Most of the light is passed directly through the grating $\left(\theta_{t 0}\right)$ and only slightly into the

Address all correspondence to W. Monty Reichert. Tel: (919) 660-5151; Fax: (919) 684-4488; E-mail: reichert@acpub.duke.edu higher order modes. It is possible to choose a grating period $(\Lambda)$, etch depth $(d)$, and profile to maximize intensity in a particular diffraction order. Our gratings have been designed and fabricated to couple through the substrate at an oblique angle of incidence and match their first diffraction order with the zeroeth order of an IOW.

Unlike other more time-consuming and expensive fabrication methods, such as direct-etch electron beam ${ }^{10}$ and phase-shift ${ }^{11}$ lithographies, our gratings are photolithographically produced, requiring only direct masked exposure, chemical development, and etching of photoresist deposited on the substrate. The ease and quantity of production are almost equivalent to embossing, ${ }^{12}$ yet offer the potential for creating sharper gratings permanently etched into the sensor.

This paper describes in detail the photolithographic technique for producing $0.7-\mu \mathrm{m}$, rectangularly justified diffraction gratings at three different etch depths. Coupling efficiencies of grating- and prism-coupled IOWs were compared to evaluate the goodness of grating design. The most efficient grating-coupled IOW was tested against a prismcoupled IOW in a simple fluoroaffinity assay. The responses of both methods were sensitive yet both 


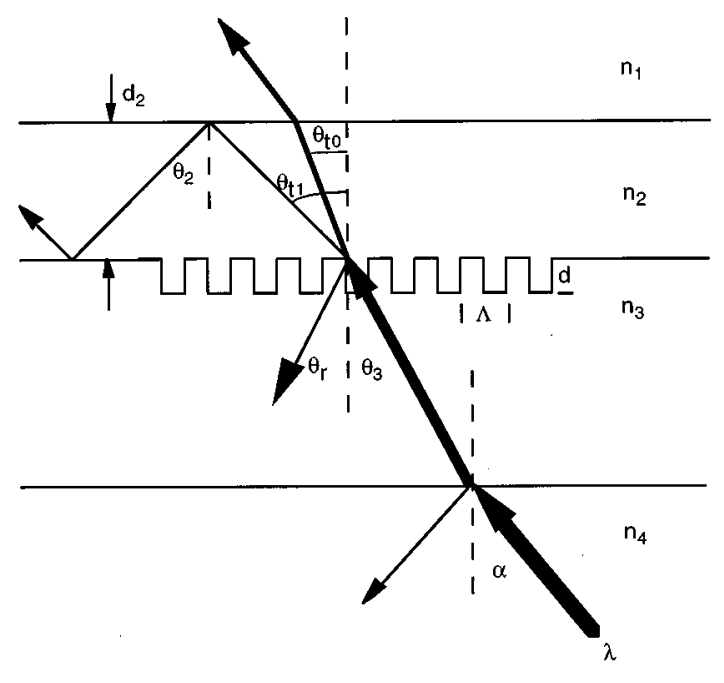

Fig. 1 Schematic of the buried grating used to couple light into an IOW. Light incident at an angle $\theta_{3}$ on the grating surface (period $=\Lambda$, etch depth $=d$ ) is reflected $\left(\theta_{r}\right)$, transmitted $\left(\theta_{t_{0}}\right)$, or diffracted $\left(\theta_{t_{1}}\right)$. Light is coupled into a guided mode when the propagation angle supported by the waveguide equals the diffraction angle $\left(\theta_{t_{1}}=\theta_{2}\right)$.

displayed signs of intra- and interassay variability which must be addressed in future generations of the sensor.

\section{THEORY}

The theory of integrated optics and grating couplers is well established. ${ }^{13}$ Briefly, referring to Figure 1, coupling from the substrate into the guided mode through the grating occurs when the plane wave direction departing the grating $\left(\theta_{t_{1}}\right)$ matches the plane wave direction of the guided mode $\left(\theta_{2}\right)$. Using the relationship for constructive interference at a grating interface and Snell's law at the substrate interface, the guiding angle $\theta_{2}$ can be related to the incident angle $\alpha$ as

$$
\Lambda\left(n_{2} \sin \theta_{2}-n_{4} \sin \alpha\right)=p \lambda
$$

where $\Lambda$ is the grating period, $n_{2}$ and $n_{4}$ are the refractive indices of the guiding and incident mediums i.e., the waveguide and air, $p$ is an integer corresponding to the grating diffraction order, and $\lambda$ is the wavelength. The waveguide angle $\theta_{2}$ for an $\mathrm{SiON}$ IOW on a quartz substrate and water superstrate is $83.6 \mathrm{deg}$ and is found by solving the following eigenvalue equation ${ }^{14}$

$$
2 k_{2} d_{2}-2 \tan ^{-1}\left(k_{1} / k_{2}\right)-2 \tan ^{-1}\left(k_{3} / k_{2}\right)=2 m \pi
$$

where $d_{2}$ is the IOW thickness, $m$ is an integer multiple corresponding to the IOW mode and for transverse electric polarization, the constant $k_{i}$ is given by

$$
k_{i}=\frac{2 \pi}{\lambda} \sqrt{n_{2}^{2} \sin ^{2} \theta_{2}-n_{i}^{2}}
$$

where $i=1,2,3$. Currently, the resolution of our photolithographic process is $0.7 \mu$; using this constraint and $632.8 \mathrm{~nm}$ light, the first diffracted order and therefore the guided mode is coupled at $38.03 \mathrm{deg}$ from the substrate normal.

\section{MATERIAls AND Methods}

\subsection{GRATING FABRICATION}

Quartz wafer substrates (Hoya, Woodcliff Lake, New Jersey, QZ 4W55-325-UP) measuring $100 \mathrm{~mm}$ in flat length and $0.5 \mathrm{~mm}$ in thickness were cleaned at room temperature in a $6 \%$ solution of hydrogen peroxide in sulfuric acid. The wafers received a hexamethyldisilazane (HMDS) vacuum vapor prime. They were then spin coated with photoresist (Shipley SNR 200, Massachusetts) at a rate of 4200 rpm for $1 \mathrm{~min}$, producing a film approximately 0.7 $\mu \mathrm{m}$ thick, and soft baked at $100^{\circ} \mathrm{C}$ for $1 \mathrm{~min}$. Negative resist, exposed to ultraviolet light (248-nm KrF excimer laser, Laser Stepper GCA-ALS, Tewksbury, Massachusetts) with a chrome-quartz mask (DuPont, Kokomo, Indiana) at a dose of $20 \mathrm{~mJ} / \mathrm{cm}^{2}$, was used to define the $0.7-\mu$ structures. The wafers received a postexposure bake at $130^{\circ} \mathrm{C}$ for $90 \mathrm{~s}$. Photoresist was developed in a solution of Shipley MF 312 at a normalization of 0.17 and then spun dry. A 60 -s $100^{\circ} \mathrm{C}$ postdevelopment bake was performed and the quartz was reactive ion etched (8110 reactive ion etcher, AME, Santa Clara, California) with $\mathrm{O}_{2}$ and $\mathrm{CHF}_{3}$ gases at an etch rate of $450 \AA / \mathrm{min}$. Etch times of 13.33, 17.77, and $22.22 \mathrm{~min}$ were employed to produce grating etch depths of $0.6,0.8$, and $1.0 \mu$. Residual photoresist was removed by $\mathrm{O}_{2}$ plasma.

\subsection{WAVEGUIDE FABRICATION}

Thin-film SiON waveguide layers were formed on the grating-etched quartz wafers in a process similar to that described by Walker et al. ${ }^{15}$ Briefly, the wafers were introduced into a plasma-enhanced chemical vapor deposition chamber (Texas Instruments) operating at $300^{\circ} \mathrm{C}, 50 \mathrm{~W}$, and 1.25 torr. The gases used were $\mathrm{SiH}_{4}$ silane, nitrogen, ammonia, and nitrous oxide. SiON films were produced at a deposition rate of approximately $590 \AA / \mathrm{min}$ for $25.42 \mathrm{~min}$, yielding a film thickness of $1.5 \mu \mathrm{m}$. The resulting waveguide wafers were diced (Disco $\mathrm{DAD}-2 \mathrm{H} / 6)$ into three rectangular pieces measuring approximately $1 \times 3$ inches.

\subsection{WAVEGUIDE CHARACTERIZATION AND EFFICIENCY MEASUREMENTS}

Prism-coupled waveguides were characterized for refractive index and thickness as already described. ${ }^{15}$ Grating waveguides were characterized in a similar fashion. Briefly, incoupling angles 
for each IOW-guided mode were measured by mounting the IOW on a goniometer (Picker X-Ray) and tuning the incident angle of a 2-mW He:Ne laser $(632.8 \mathrm{~nm}, 10 \mathrm{~mW}$ maximum, Melles-Griot, Uniphase, Manteca, California) until a guided mode was established. Thicknesses were determined by converging on a solution to the eigenvalue equation [Eq. (2)] using an IOW refractive index guess $\left(n_{2}\right.$ $=1.53$ ) and substrate and superstrate refractive indices $\left(n_{3}=1.46\right.$ and $\left.n_{1}=1.00\right)$. Separate computer programs were used to account for differences in converting measured incident angles to guiding angles through their respective couplers.

Efficiencies for both prisms and gratings were measured by using the method of Dakss et al. ${ }^{16}$ IOWs were mounted on a rotation stage and the intensities of the incident, reflected, and transmitted beams were recorded as the waveguide entered and passed through a guided mode. Since power contained in the guided mode was difficult to measure, the incident minus transmitted plus reflected intensities were used to approximate this quantity. Efficiencies were determined by ratioing the guided mode intensity with the incident intensity and in the case of grating IOWs, normalized by the prism efficiency.

\subsection{GRATING WAVEGUIDE SAMPLE CELL}

The sample cell (refer to Figure 2) employed was a modified version of a prism sample cell reported previously. ${ }^{9}$ Quarter-inch stock aluminum was cut into a $1 \times 3$-inch rectangle. Four flow ports were drilled into the aluminum at 1-inch diagonals from the corners. The entire piece was anodized flat black (Precision Alloys, Raleigh, North Carolina) and fit with hose connectors (Small Parts, Inc., Miami Lakes, Florida). A rectangular, two-channel gasket was formed from 0.02-inch medical-grade NRV silastic sheeting (Dow Corning Corp., Midland, Michigan). Each channel, which ran lengthwise from one flow port to another, was 0.25 $\times 2$ inches, producing a sample cell volume of approximately $160 \mu \mathrm{l}$.

\subsection{ASSAY FOR CYANINE DYE-LABELED AVIDIN}

Cyanine dye (Cy5, Research Organics, Inc., Cleveland, Ohio) was used to label avidin (Sigma Chemical Co., St. Louis, MO) with 3 to 4 dyes per protein. Briefly, $2 \mathrm{mg}$ of avidin were dissolved into a $\mathrm{pH} 8.3$ bicarbonate buffer and added to a vial containing the dye. This mixture was allowed to incubate at room temperature for $30 \mathrm{~min}$, vortexing every 10 min. The protein was separated from free dye using a PD-10 column (Pharmacia Biotech, Piscataway, New Jersey) and reconstituted in a phosphatebuffered saline solution (PBS, Sigma Chemical Co.). Stock concentration was determined to be approximately $2 \times 10^{-5} \mathrm{M}$ by measuring absorbance (Spectronic Genesis Spectrophotometer, Milton Roy,

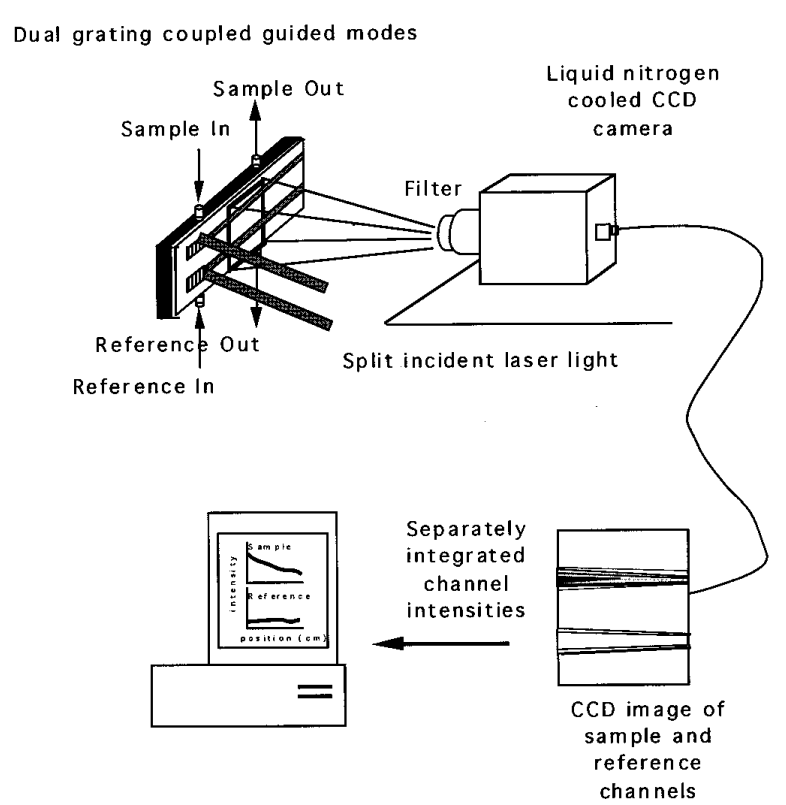

Fig. 2 Apparatus used in dual-channel affinity assay. A CCD collects fluorescence from the IOW surface that has been filtered by a bandpass interference filter and is subsequently sent to a computer for analysis.

Rochester, New York) at $280 \mathrm{~nm}\left(\epsilon_{280}^{0.1 \% \text { avidin }}\right.$ $=96,000 \mathrm{M}^{-1} \mathrm{~cm}^{-1}$ ) for protein and $6 \times 10^{-6} \mathrm{M}$ at $653 \mathrm{~nm}\left(\epsilon_{653}^{\mathrm{Cy} 5}=250,000 \mathrm{M}^{-1} \mathrm{~cm}^{-1}\right)$ for the dye. This was subsequently diluted serially into a background solution containing $1 \times 10^{-7} M$ Cy5-BSA $\left(\epsilon_{280}^{0.1 \% \mathrm{BSA}}=44,220 \mathrm{M}^{-1} \mathrm{~cm}^{-1}\right)$ labeled in a similar fashion to give 1-ml samples in the range of 1 $\times 10^{-15} \mathrm{M}$ to $1 \times 10^{-8} \mathrm{M}$; i.e., each avidin "analyte" dilution was premixed with a $1 \times 10^{-7} M$ Cy5-BSA "tracer" solution prior to measurement. A dualchannel assay ${ }^{9}$ was performed on both a grating and prism-coupled $\mathrm{SiON}$ waveguide preadsorbed for $2 \mathrm{hr}$ at room temperature with $2 \mathrm{mg} / \mathrm{ml}$ biotinlabeled BSA (Sigma Chemical Co.).

The apparatus used to collect images in both prism and grating configurations is shown in Figure 2. Each injected sample or reference solution was incubated with the waveguide surface for 7 min, followed by image collection over a 10-s integration time. Sample and reference images were collected simultaneously. The sequence of images for each assay consisted of (1) collecting a "buffer" background of PBS in both reference and sample channels, (2) collecting a "tracer" background in both reference and sample channels, and (3) collecting serial images of increasing analyte/tracer mixtures in the sample channel and tracer solution in the reference channel.

A total of six assays were conducted: three for prism-coupled waveguides and three for gratingcoupled waveguides. Seven analyte concentrations were tested per assay $\left(10^{-15}, 10^{-14}, 10^{-13}, 10^{-12}\right.$ and $10^{-11}, 10^{-10}$, and $10^{-9} M$ avidin). At the end of 


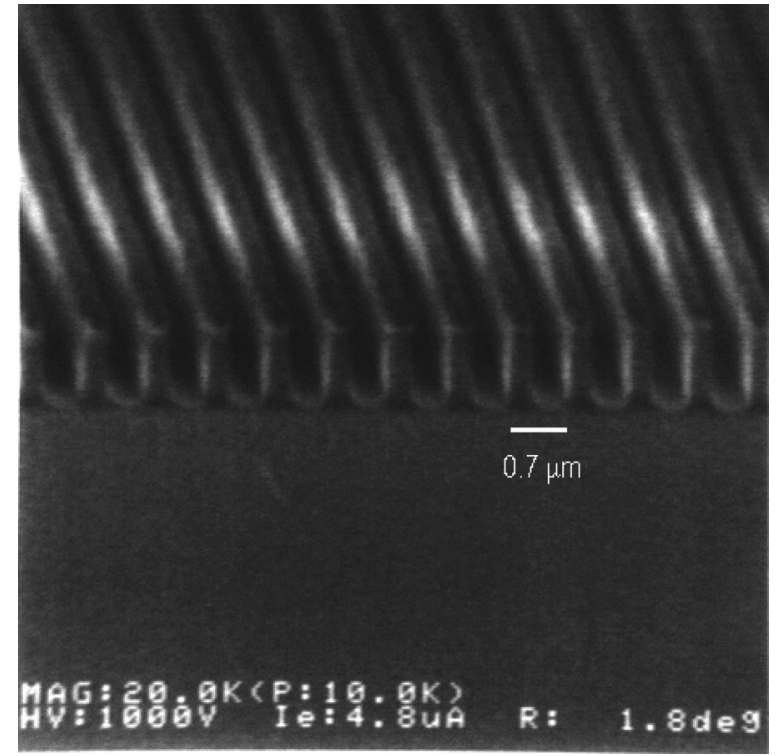

Fig. 3 SEM of photolithographically defined grating. The period between grooves is $0.7 \mu \mathrm{m}$ and the etch depth is $1.0 \mu \mathrm{m}$.

each assay, a 10-nM solution of Cy5-Av was injected to saturate the sample channel surface, producing a "saturation" image for normalization purposes. Images were converted to pixel intensities with NIH Image. Background-subtracted sample intensities were used to construct binding curves for each experiment.

\section{RESULTS}

\subsection{WAVEGUIDE CHARACTERIZATION}

The final form of an etched grating is shown in Figure 3; this particular scanning electron micrograph (SEM) is for a $0.7-\mu \mathrm{m}$ period grating having an etch depth of $1 \mu \mathrm{m}$. Rectangular profiles are evident except for a slight curvature in each trough. Although the etch process should be perfectly anisotropic, occurring only in the vertical direction, some lateral etching appears, giving the tapered appearance. The duty cycle of the grating structure is $50 \%$, i.e., the distance between crests is the same as the distance between troughs. The gratings have been etched deeper than they are wide, giving an aspect ratio (etch depth/grating period) of 1.4. SEMs were not obtained for the 0.8- and 0.6- $\mu$ m-deep gratings. Table 1 lists further measured properties of prismand grating-coupled $\mathrm{SiON}$ IOWs in the presence of a water superstrate.

Prisms were determined to couple $85 \%$ of incident light, almost 4 percentage points above their theoretical maximum of $81.4 \% .{ }^{17}$ This overestimate most likely arises from our inability to measure light lost to scatter and oblique reflections. To account for this, we ratio grating values to prism values in order to obtain a relative degree of coupling for the gratings. Although this does not give an exact value for coupling efficiencies, it is a good indicator of grating performance compared with prism coupling. There was not a significant difference in relative coupling efficiency of the 0.6- and $0.8-\mu \mathrm{m}$ deep gratings: $19.21 \pm 4.75 \%$ and $19.61 \pm 7.56 \%$, respectively. The $1.0-\mu \mathrm{m}$-deep gratings significantly improved in efficiency to $55.29 \pm 4.25 \%$. Although the trend in the data is correct, one would predict a greater increase in efficiency between $0.6-\mu \mathrm{m}$ and 0.8 - $\mu$ m-deep gratings-ideally this trend would be proportional to the increase in etch depth. It is likely that the actual efficiency of the $0.8-\mu$ m-deep gratings lies somewhere between 25 and $35 \%$. The failure of our observations to follow this trend and the elevated standard deviation of the $0.8-\mu \mathrm{m}$ grating measurement suggest that an experimental error arose from inaccuracies in the optical alignment or signal collection method.

\subsection{ASSAYS}

Fluorescent images of the reference and sample channels were collected in triplicate for a series of avidin analyte concentrations over the $1 \mathrm{fM}$ to $10 \mathrm{nM}$ range using both prism- and gratingcoupled waveguides. The dose-response curves for each set of assays are shown in Figure 4. A twocomponent, three-parameter model was used to fit the data. Briefly, this model assumes that there are two noncooperative populations of receptors on the

Table 1 Physical characteristics of prism- and grating-coupled SiON waveguides measured with a water superstrate at $633 \mathrm{~nm}$.

\begin{tabular}{|c|c|c|c|c|c|c|}
\hline & \multicolumn{2}{|c|}{ Prism } & \multicolumn{4}{|c|}{ Grating } \\
\hline & Measured $^{a}$ & Expected & Measured & Expected & \multicolumn{2}{|c|}{ Efficiency $(\eta)$ data } \\
\hline$\alpha(m=0)$ & $19.0 \pm 0.2 \mathrm{deg}$ & $19.3 \mathrm{deg}$ & $37.8 \pm 0.8 \mathrm{deg}$ & $38.0 \mathrm{deg}$ & Depth & $\%$ Prism $\eta$ \\
\hline$d_{2}(\mu \mathrm{m})$ & $1.3 \pm 0.1$ & 1.5 & $1.5 \pm 0.2$ & 1.50 & $0.6 \mu \mathrm{m}$ & $19.2 \pm 4.8$ \\
\hline$n_{2}$ & $1.53 \pm 0.00$ & 1.53 & $1.53 \pm 0.01$ & 1.53 & $0.8 \mu \mathrm{m}$ & $19.6 \pm 7.6$ \\
\hline$\Lambda(\mu \mathrm{m})$ & $N / A^{b}$ & $\mathrm{~N} / \mathrm{A}$ & 0.7 & 0.7 & $1.0 \mu \mathrm{m}$ & $55.3 \pm 4.3$ \\
\hline
\end{tabular}




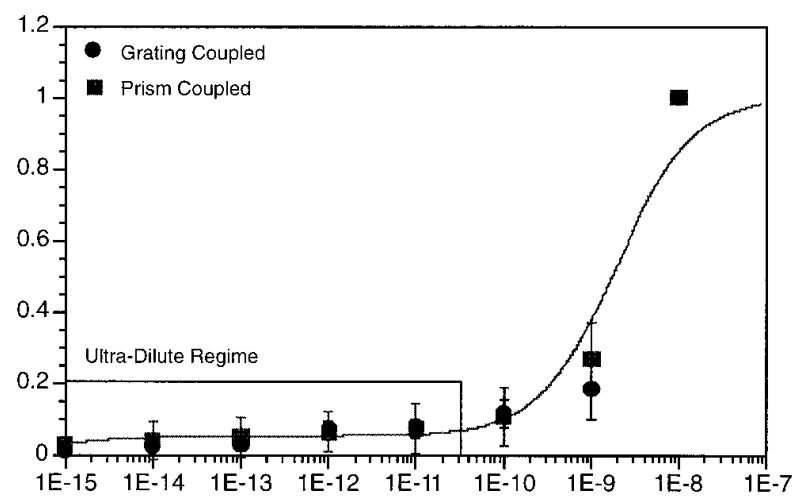

Fig. 4 Dose-response curves generated from prism- (squares) and grating- (circles) coupled IOW affinity binding experiments $(n=3)$. The boxed region defines the ultradilute regime studied in this work. The solid line is a two-component, three-parameter Langmuir fit.

surface. One fraction $\left(r_{1}\right)$ of the population binds with unusually high, multivalent affinity $\left(K_{1}\right)$ and the other $\left(1-r_{1}\right)$ with lower, monovalent affinity $\left(K_{2}\right)$, so that the overall response is expressed as the sum of the two terms

$$
\theta=r_{1} \frac{K_{1} C}{1+K_{1} C}+\left(1-r_{1}\right) \frac{K_{2} C}{1+K_{2} C}
$$

where $\theta$ is the total fraction of bound analyte and $C$ is the bulk concentration. Table 2 shows the curvefitting parameters of intra- and interassay data. The goodness of fit in all cases was at least $95 \%$. Although discrete changes in intensity were measured at low concentrations $\left(<10^{-11} M\right)$, one case in each intraassay group predicted $0 \%$ multivalent binding while the remaining cases predicted at least $5 \%$. Both intra- $(\mathrm{CV}>70 \%)$ and interassay groups showed an order of magnitude difference in the high affinity rate constant $\left(K_{1}\right)$, which varied from as low as $2 \times 10^{12} \mathrm{M}^{-1}$ up to $3 \times 10^{15} \mathrm{M}^{-1}$. These results suggest that the model is extremely sensitive to intensity fluctuations, which may account for some or all of the signal at low concentrations. It is encouraging that the low-affinity rate constant $K_{2}$ was in good agreement across all experiments at 5 $\times 10^{8} M^{-1}$, correlating well with our previous work. ${ }^{9}$ Clearly, optimization of both apparatus and surface chemistry is necessary in future generations of the grating-coupled sensor if the lowconcentration regime is to be adequately studied.

\section{DISCUSSION}

The ultradilute regime, boxed range of Figure 4 covering the $1 \mathrm{fM}$ to $10 \mathrm{pM}$ concentrations is the region of interest for this study. The tracercorrected sample channel images (i.e., tracer image subtracted) for each ultradilute measurement are given in Figure 5. These images have been inverted and converted from color to gray scale. Averaged over a $15 \times 100$-pixel area, the mean intensities of prism-coupled fluorescent images were at least twofold greater than the corresponding gratingcoupled images. However, when the normalized mean intensities are plotted against concentration, both predict a semilogarithmic response (also already reported ${ }^{9}$ ).

Although theoretically nonlinear over the doseresponse curve, Figure 6 clearly shows that a semilogarithmic fit to the data works well in the ultradilute concentration range. The solid lines are linear best fits to the data, described in Table 3. Prismand grating-coupled methods yielded slopes ranging from $0.4 \times 10^{-2}$ to $2.6 \times 10^{-2}$, with five of the six assays having an error in slope of $26 \%$ or less. Viewed independently, all of the prism-coupled assays produced linear fits that were extremely sig-

Table 2 Two-component, three-parameter values for individual and grouped experiments.

\begin{tabular}{|c|c|c|c|c|c|c|c|c|c|c|}
\hline \multirow[b]{3}{*}{ Parameter } & \multicolumn{8}{|c|}{ Intraassay data } & \multicolumn{2}{|c|}{ Interassay data } \\
\hline & \multicolumn{3}{|c|}{ Prism } & \multirow[b]{2}{*}{$\mathrm{CV}$} & \multicolumn{3}{|c|}{ Grating } & \multirow[b]{2}{*}{$\mathrm{CV}$} & \multirow{2}{*}{$\begin{array}{l}\text { Prism } \\
\text { Avg \# }\end{array}$} & \multirow{2}{*}{$\begin{array}{c}\text { Grating } \\
\text { Avg \# }\end{array}$} \\
\hline & 1 & 2 & 3 & & 1 & 2 & 3 & & & \\
\hline$r_{1}$ & 0.06 & 0.05 & 0.00 & 9.1 & 0.09 & 0.00 & 0.09 & 0.00 & 0.05 & 0.05 \\
\hline $1-r_{1}$ & 0.94 & 0.95 & 1.00 & 2.7 & 0.91 & 1.00 & 0.91 & 4.5 & 0.95 & 0.95 \\
\hline$K_{1}^{a}$ & 2.67 & 26.1 & $N / A^{b}$ & 81.4 & 0.11 & N/A & 0.02 & 69.2 & 17.0 & 1.78 \\
\hline$K_{2}{ }^{c}$ & 5.34 & 5.45 & 4.06 & 12.7 & 5.02 & 3.64 & 4.11 & 13.5 & 5.09 & 4.16 \\
\hline$r_{2}$ & 0.97 & 0.97 & 0.94 & & 0.98 & 0.96 & 0.97 & & 0.97 & 0.95 \\
\hline
\end{tabular}

a $\times 10^{14} \mathrm{M}^{-1}$.

${ }^{b} \mathrm{~N} / \mathrm{A}=$ not avialable.

c $\times 10^{8} \mathrm{M}^{-1}$ 


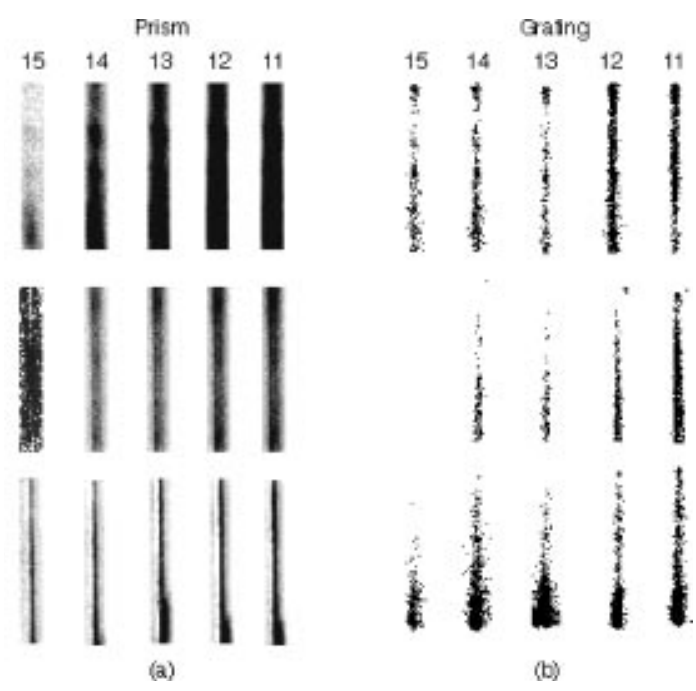

Fig. 5 Sample channel images for (a) prism and (b) grating experiments $(n=3)$ over the concentration range $1 \times 10^{-15} \mathrm{M}$ to $1 \times 10^{-11} \mathrm{M}$.

nificant $(p<0.01)$. Whereas two of the three grating-coupled assays had significant linear fits $(p<0.05)$, the remaining grating-coupled assay had a linear fit that was statistically insignificant.

An important piece of information derived from the work presented here would be to quantitatively determine how well gratings performed relative to prisms in the affinity assay. The standard for making this assessment is determination of the confidence envelope, usually the $95 \%$ confidence limit, and the resulting detection limit. In order to gather this information, the data from the three prism- and grating-coupled assays were pooled into two data sets and input into the statistical program InStat (GraphPad Software). Figure 7 is the result of this analysis. The upper and lower curved lines are the resultant $95 \%$ confidence envelopes. The solid straight lines are the linear best fits to the data. Using the technique described by Lin et al., ${ }^{18}$ the detection limit was determined from extrapolation of the confidence envelope (see dashed lines). In both cases, the $95 \%$ confidence detection limit was deter-

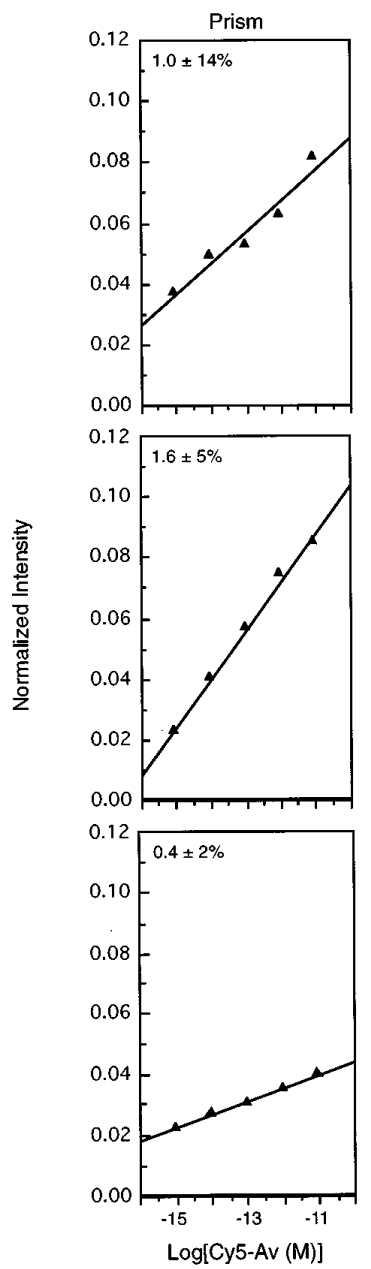

(a)
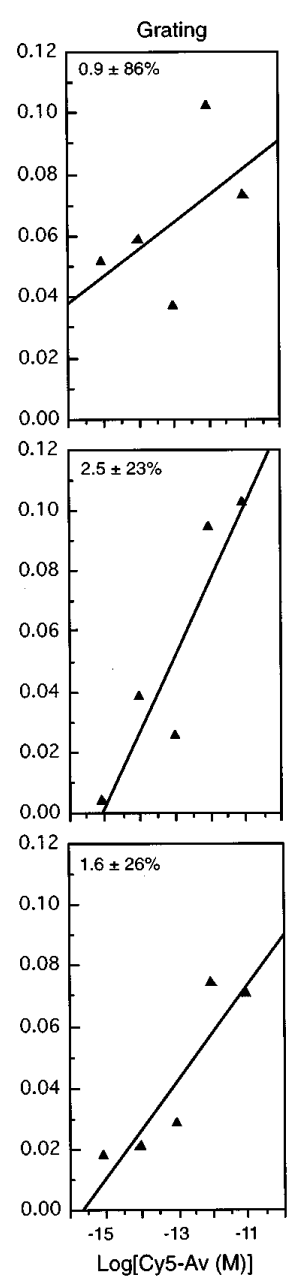

(b)
Fig. 6 Log-normal plot comparing the mean pixel intensity versus analyte concentration of (a) prism- and (b) grating-coupled sensors $(n=3)$. The numbers in the upper left-hand corner of each image are the slopes $\left(\times 10^{2}\right)$ of best-fit lines to the data points.

mined to be on the order of $0.1 \mathrm{pM}$. It is interesting that the linear best fits to the pooled prism and grating data produced identical sensitivities (Student's $t$-test) and nearly identical standard errors,

Table 3 Linear regression to individual prism and grating data over the ultradilute regime.

\begin{tabular}{|c|c|c|c|c|}
\hline \multirow[b]{2}{*}{ No. } & \multicolumn{2}{|c|}{ Prism } & \multicolumn{2}{|c|}{ Grating } \\
\hline & Slope \pm percent error & $p$ Value & Slope \pm percent error & $p$ Value \\
\hline 1 & $1.03 \times 10^{-2} \pm 14$ & $6.0 \times 10^{-3 a}$ & $8.80 \times 10^{-3} \pm 86$ & $3.3 \times 10^{-1}$ \\
\hline 2 & $1.59 \times 10^{-2} \pm 5.0$ & $0.3 \times 10^{-3 a}$ & $2.54 \times 10^{-2} \pm 23$ & $2.4 \times 10^{-2 b}$ \\
\hline 3 & $4.30 \times 10^{-2} \pm 2.3$ & $2.7 \times 10^{-5 a}$ & $1.59 \times 10^{-2} \pm 26$ & $3.1 \times 10^{-2 b}$ \\
\hline
\end{tabular}

a Significant to $99 \%$ confidence limit $(p<0.01)$.

b Significant to $95 \%$ confidence limit $(p<0.05)$. 


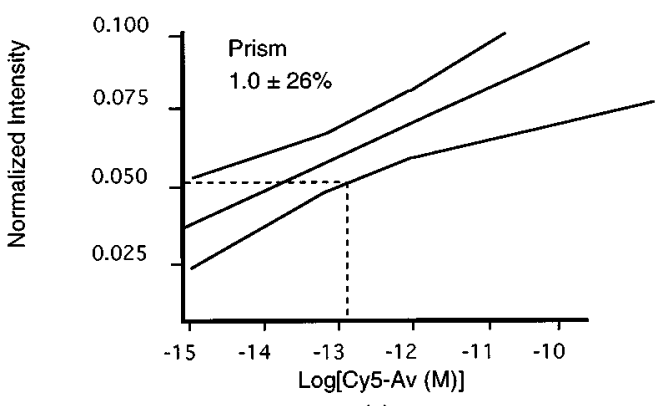

(a)

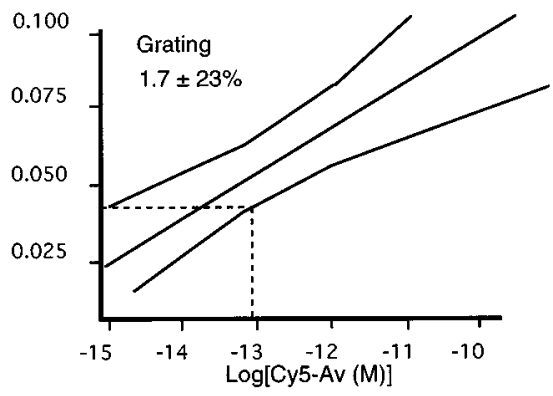

(b)

Fig. 7 Comparison of grouped prism and grating data. The upper and lower curved lines are the resultant $95 \%$ confidence envelopes. The solid straight lines are the linear best fit to the data. The numbers in the left-hand corner are the best-fit slopes $\left(\times 10^{2}\right)$ and their errors. The detection limit was determined from extrapolation of the confidence envelope (see dashed lines) to be on the order of $0.1 \mathrm{pM}$.

and both data sets fit a straight line with greater than $99 \%$ confidence $(p<0.01)$. This information is summarized in Table 4 . Viewed individually, however, both grating and prism-coupled sensors were clearly capable of detecting a $10^{-15} \mathrm{M}$ analyte above background; however, it is also clear that the grating-coupled sensor exhibited more scatter in the data.

Per run variability arises from scatter and bulk contributions; this is a prevalent problem with grating sensors, which tend to scatter more incident light than prisms. Run-to-run changes may arise from the variable number of binding sites immobilized on each transducer and from variations in coupling efficiency. These effects can be controlled in future generations of the sensor by (1) maintaining the total number of binding sites between experiments, (2) engineering an alignment system to ensure identical placement of the transducer, and (3) reducing scattered light introduced into the sensing area by improving the grating design to boost efficiency and possibly adding optical stops down line from the coupling area.

Interesting questions that arise from this ultradilute detection are "Why does one observe affinity binding at these very low concentrations?" "Why is this response semilogarithmic?" "Is this response confined to high-affinity systems like avidin/ biotin?" Possible explanations have been offered for this non-Langmuirian behavior': (1) the system is not in equilibrium after the short binding times
$(<10 \mathrm{~min})$ and (2) there are possibly multivalent interactions taking place between receptor and ligand. Others have observed similar effects with their sensors that corroborate our observations. Herron et al. ${ }^{19}$ proposed that multivalent tracer molecules stabilized receptor-ligand complex formation at the surface, further increasing detection below that predicted by the Langmuir model. Interestingly, they observe semilogarithmic responses in lower affinity systems, such as antigen/antibody. Schlatter et al. ${ }^{20}$ suggested implementation of a more rigorous binding model when receptor and ligand have the potential to interact multivalently, as in the case of avidin and biotin. Clearly, these effects warrant further elucidation.

\section{CONCLUSIONS}

Photolithography was used to produce gratings with a $0.7-\mu$ pitch and $1.0-\mu$ etch depth. When used as optical couplers for thin-film IOWs, these gratings were roughly half as efficient as prisms in coupling 632.8-nm light. In a set of comparative IOW fluoroaffinity assays, two of the three experiments with each sensor detected analyte above background in the ultradilute regime of $1 \mathrm{fM}$ to $10 \mathrm{pM}$. All measurements exhibited the semilogarithmic response that was noted previously. ${ }^{9}$ The gratingcoupled measurements were less precise, run to run, than prism-coupled IOW-FIA measurements, as indicated by the uncertainty of an arbitrary lin-

Table 4 Linear regression and comparison of slopes for pooled prism and grating data over the ultradilute regime.

\begin{tabular}{lcccc}
\hline & $\begin{array}{c}\text { Slope } \pm \text { percent } \\
\text { error }\end{array}$ & $p$ Value & Detection limit & $\begin{array}{c}\text { Statistical comparison of grating } \\
\text { and prism slopes using Student's } \\
t \text { test }\end{array}$ \\
\hline Prism & $1.00 \times 10^{-2} \pm 26$ & $2.4 \times 10^{-3 a}$ & $8.0 \times 10^{-12} \mathrm{M}$ & $t=0.929 ; t_{0.05(2), 26}=2.056$ \\
Grating & $1.67 \times 10^{-2} \pm 23$ & $8.6 \times 10^{-4 a}$ & $1.2 \times 10^{-13} \mathrm{M}$ & $\begin{array}{c}p \text { value: } 0.3835 ; \text { no significant } \\
\text { difference between slopes }\end{array}$ \\
\hline
\end{tabular}

a Significant to $99 \%$ confidence limit $(p<0.01)$. 
ear regression. To judge reproducibility, the three grating-coupled and three prism-coupled measurements were pooled into two data sets, both of which fit a straight line with extreme significance $(p<0.01)$. Viewed in this manner, the grating- and prism-coupled IOWs yielded similar sensitivity, detection limit, and percent error. Although both approaches detected samples with femtomolar concentrations above background, the run-to-run variability, more so than the per run variability, appears to be the primary source of uncertainty in the detection limit.

\section{Acknowledgments}

We gratefully acknowledge support from National Institutes of Health Grant HL 32132, National Science Foundation (NSF) Grant BES-9402355, and the NSF/ERC Center for Emerging Cardiovascular Technologies. Appreciation is extended to Jim Herron and Doug Christensen at the University of Utah for helpful discussions on assay protocol and physical optics, and to anonymous reviewers for many insightful comments. Portions of this paper report technology licensed to HCP Diagnostics of Salt Lake City, Utah.

\section{REFERENCES}

1. A. N. Sloper, J. K. Deacon, and M. T. Flanagan, "A planar indium phosphate monomode waveguide evanescent field immunosensor," Sens. Actuat. B1, 589-591 (1990).

2. S. W. Kang, K. Sasaki, and H. Minamitani, "Sensitivity analysis of a thin film optical waveguide biochemical sensor using evanescent field absorption," Appl. Opt. 32, 3544-3549 (1993).

3. J. J. Ramsden, S. Y. Li, E. Heinzle, and J. E. Prenosil, "Optical method for measurement of number and shape of attached cells in real time," Cytometry 29, 97-102 (1995).

4. N. J. Fell and P. W. Bohn, "In situ evaluation of diffusion mechanisms in thin polystyrene films used as integrated optical structures," Appl. Spectrosc. 45, 1085-1092 (1991).

5. B. A. Bolton and J. R. Scherer, "Raman spectra and water absorption of bovine serum albumin," J. Phys. Chem. 93, 7635-7640 (1989).

6. S. B. Mendes, L. Li, J. Burke, J. E. Lee, D. R. Dunphy, and S. S. Saavedra, "Broad-band attenuated total reflection spec- troscopy of a hydrated protein film on a single mode planar waveguide," Langmuir 12, 3374-3376 (1996).

7. L. Yang, S. S. Saavedra, and N. R. Armstrong, "Sol-gel based, planar waveguide sensor for gaseous iodine," Anal. Chem. 68, 1834-1841 (1996).

8. T. E. Plowman, S. S. Saavedra, and W. M. Reichert, "Planar integrated optical methods for examining thin films and their surface adlayers," Biomaterials (in press).

9. T. E. Plowman, H. K. Wang, D. A. Christensen, J. N. Herron, C. R. Peters, and W. M. Reichert, "Femtomolar sensitivity using a channel-etched thin film waveguide fluoroimmunosensor," Biosensors Bioelectronics 11, 149-160 (1996).

10. P. Blair, M. R. Taghizadeh, W. Parkes, and C. D. W. Wilkinson, "High-efficiency binary fan-out gratings by modulation of a high-frequency carrier grating," Appl. Opt. 34, 24062413 (1995).

11. M. D. Levenson, N. S. Viswanathan, and R. A. Simpson, "Improving resolution in photolithography with a phaseshifting mask," IEEE Trans. Elec. Dev. ED-29, 1828-1836 (1982).

12. Ph. M. Nellon and W. Lukosz, "Integrated optical input grating couplers as direct affinity sensors," Biosensors Bioelectronics 8, 129-147 (1993).

13. D. L. Lee, Electromagnetic Principles of Integrated Optics, John Wiley, New York (1986).

14. S. S. Saavedra and W. M. Reichert, "Integrated optical attenuated total reflection spectrometry of aqueous superstrates using prism-coupled polymer waveguides," Anal. Chem. 62, 2251-2256 (1990).

15. D. S. Walker, W. M. Reichert, and C. J. Berry, "Corning 7059, silicon oxynitride, and silicon dioxide thin-film integrated optical waveguides: in search of low-low, nonfluorescent reusable glass waveguides," Appl. Spectrosc. 46, 1437-1441 (1992).

16. M. L. Dakss, L. Kuhn, P. F. Heidrich, and B. A. Scott, "Grating coupler for efficient excitation of optical guided waves in thin films," Appl. Phys. Lett. 16(12), 523-525 (1970).

17. T. Tamir and S. T. Peng, "Analysis and design of grating couplers," Appl. Phys. 14, 235-254 (1977).

18. J. N. Lin, W. C. Mahoney, A. A. Luderer, R. A. Brier, T. W. Sharp, and V. A. McGuire, "Ultra sensative evanescent wave fluoro-immunosensors using polystyrene integrated lens optical fiber," Proc. SPIE, Vol. 2138, pp. 204-215 (1994).

19. J. Herron, K. Caldwell, D. Christensen, S. Dyer, V. Hlady, P. Huang, V. Janatova, H. Wang, and A. Wei, "Fluorescent immunosensors using planar waveguides," Adv. Fluorescent Sensing Technol. 1885, 28 (1993).

20. D. Schlatter, R. Barner, Ch. Fattinger, W. Huber, J. Hubscher, J. Hurst, H. Koller, C. Mangold, and F. Muller, "The difference interferometer: application as a direct affinity sensor," Biosensors Bioelectronics 8, 109-116 (1993). 